\title{
Farewell to oligoastrocytoma: response to letters
}

\author{
Felix Sahm • Andreas von Deimling
}

Received: 16 October 2014 / Revised: 4 November 2014 / Accepted: 4 November 2014 / Published online: 9 November 2014

(C) Springer-Verlag Berlin Heidelberg 2014

A proposal to part with diagnosing a tumor entity recognized for nearly a century naturally will be critically questioned. Such happened following our paper entitled "Farewell to Oligoastrocytomas" [3]. Before commenting on the responses by Wilcox et al. and Huse et al. [1, 4], we would like to point at the current practice. The frequency of the diagnosis "oligoastrocytoma" varies from essentially zero to up to $80 \%$ of the diagnoses made for diffusely infiltrating gliomas-depending on the diagnosing institution. As a consequence, our clinical partners appear less interested in our differentiation among astrocytoma, oligoastrocytoma or oligodendroglioma - they ask: "Does it carry a $1 \mathrm{p} / 19 \mathrm{q}$ codel or does it not". This association has indeed been shown in many clinical studies to be of significant clinical relevance. This tight association of clinical and molecular genetic parameters is a stringent argument for an attempt to separate the "oligoastrocytomas" primarily on molecular grounds into two subgroups. Done so, there is close to nothing left to distinguish these subgroups from astrocytoma on the one and oligodendroglioma on the other side. Of course, and likely no tumor entity can be exempted here, we will detect a small fraction of tumors demonstrating overlapping molecular features.

Wilcox et al. present in their response letter two cases and very convincingly demonstrate in one of them such overlapping molecular features and we congratulate this nice work. The evidence provided for their second case appears to rely mainly on immunohistochemistry presented for single cells only and we think it is in need for further analysis to fully support the claim.
Huse et al. also identified a case with two components, one compatible with astrocytoma and one with oligodendroglioma.

Nevertheless, our paper and the responses of our colleagues exemplify a central problem to all tumor classification systems. Where do we draw a line to obtain clearly defined diagnostic groups with direct clinical implication without neglecting the existence of rare cases not matching? The International Society of Neuropathology-Haarlem Consensus Guidelines [2], prepared as a basis for the upcoming revision of WHO classification of brain tumors, provide a direction: clear and stringently defined tumor entities will be preferred and small groups of tumors currently not matching these entities will be accepted.

\section{References}

1. Huse JT, Diamond EL, Wang M, Rosenblum MK (2015) Mixed glioma with molecular features of composite oligodendroglioma and astrocytoma: a true "oligoastrocytoma"? Acta Neuropathol. doi:10.1007/s00401-014-1359-y

2. Louis DN, Perry A, Burger P et al (2014) International society of neuropathology-haarlem consensus guidelines for nervous system tumor classification and grading. Brain Pathol 24:429-435

3. Sahm F, Reuss D, Koelsche C et al (2014) Farewell to oligoastrocytoma: in situ molecular genetics favor classification as either oligodendroglioma or astrocytoma. Acta Neuropathol 128:551-559

4. Wilcox P, Li CCY, Lee MYT et al (2015) Oligoastrocytomas: throwing the baby out with the bathwater? Acta Neuropathol. doi:10.1007/s00401-014-1353-4
F. Sahm $\cdot$ A. von Deimling $(\bowtie)$

Department of Neuropathology, Institute of Pathology, Ruprecht-

Karls-University Heidelberg, and German Cancer Consortium

(DKTK), Clinical Cooperation Unit Neuropathology, German

Cancer Research Center (DKFZ), Im Neuenheimer Feld 224,

69120 Heidelberg, Germany

e-mail: andreas.vondeimling@med.uni-heidelberg.de 